\title{
The stigma of mental illness: effects of labelling on public attitudes towards people with mental disorder
}

Angermeyer MC, Matschinger H. The stigma of mental illness: effects of
labelling on public attitudes towards people with mental disorder. Acta Psychiatr Scand 2003: 108: 304-309. @ Blackwell Munksgaard 2003.

Objective: Aim of the study is to examine the impact of labelling on public attitudes towards people with schizophrenia and major depression.

Method: In Spring 2001, a representative survey was carried out in Germany involving adults of German nationality $(n=5025)$.

Results: Labelling as mental illness has an impact on public attitudes towards people with schizophrenia, with negative effects clearly outweighing positive effects. Endorsing the stereotype of dangerousness has a strong negative effect on the way people react emotionally to someone with schizophrenia and increases the preference for social distance. By contrast, perceiving someone with schizophrenia as being in need for help evokes mixed feelings and affects people's desire for social distance both positively and negatively. Labelling has practically no effect on public attitudes towards people with major depression.

Conclusion: Our findings illustrate the need for differentiation, differentiation between the different components of stigma as well as differentiation between the various mental disorders.

\section{C. Angermeyer, H. Matschinger}

Department of Psychiatry, University of Leipzig, Leipzig, Germany

Key words: stigma; labelling; attitudes; schizophrenia; major depression

Dr Matthias C. Angermeyer, Department of Psychiatry, University of Leipzig, Johannisallee 20, 04317 Leipzig. Germany

E-mail: krausem@medizin.uni-leipzig.de

Accepted for publication April 3, 2003

\section{Introduction}

There are two opposing views on the labelling of mental health problems as mental illness. From a clinical point of view it is argued that labelling provides orientation for those afflicted and their relatives. The uncertainty and false beliefs will be replaced by a better understanding of the nature of the problem. People will then know better whom to ask for help and which measures to take to overcome the problem. Sociological role theory points to another positive effect of labelling: if the mental health problem is seen as an illness, the privileges of the patient role will be granted (1), and patients will not be held responsible for their illness. All this should result in a more accepting attitude towards those suffering from mental disorder. By contrast, the labelling approach stresses the negative effects of psychiatric labelling (2). According to this theory, through labelling the negative stereotype of the mentally ill, which is still prevalent among the general public, will be triggered. This in turn will lead to increased discrimination against those suffering from mental disorder.

Empirical evidence for these two opposing views on labelling effects is equivocal. Discussing experimental studies in which the label had been manipulated, Farina (3) came to the conclusion that defining mental health problems as mental illness (instead of social problems) neither reduced stigmatization nor improved the perception of the afflicted. In a review of studies involving experimental or quasiexperimental designs assessing the relative effects of mental illness label vs. some variation of behaviour, Link et al. (4) observed that although most studies found some evidence of labelling effects, these effects had either not been significant across all the outcomes assessed or had not been in the direction of more severe labels leading to more severe rejection. However, based on a vignette experiment, the authors were able to show that labelling fosters 
high social distance among those who perceive mental patients to be dangerous.

According to Corrigan $(5,6)$ three different stigma components can be distinguished: stereotypes, prejudice, and discrimination. Stereotypes represent notions of groups of persons, in our case the mentally ill, which have collectively been agreed upon. People who are prejudiced endorse these negative stereotypes and, as a result, generate emotional reactions. Prejudice, which is basically a cognitive and affective response, leads to social discrimination, the behavioural reaction. In our study, we will examine the impact of labelling as mental illness on these three attitudinal domains.

Aims of the study

Based on data from a representative population survey, the impact of labelling as mental illness on the three stigma components identified by Corrigan will be examined. Drawing on the literature on labelling and its effect on people with mental disorder, the following hypotheses have been formulated, contrasting two main stereotypes about the mentally ill, namely that they are dangerous and that they are needy and dependent on others:

1) Respondents who label the problem depicted in the vignette as mental illness are more likely to perceive the individual as being dangerous.

2) The more respondents perceive the individual as being dangerous, the more they will react with fear.

3) Fear, in turn, will lead to an increase of the desire for social distance.

4) Respondents who label the problem as mental illness are more likely to perceive the individual as being in need for help.

5) The more the individual depicted in the vignette is perceived as being in need for help, the more people will react with pity and the less they will react with anger.

6) An increase of pity as well as a decrease of anger will lead to greater acceptance of the individual, i.e. the preference for social distance will be inversely affected.

7) The impact of labelling on attitudes should be more pronounced with schizophrenia than with major depression.

\section{Material and methods}

Sample

During May and June of 2001, a representative survey was conducted in Germany, involving persons of German nationality who were at least 18 years old and who were living in private households. The sample was drawn using a threestage random sampling procedure with electoral wards at the first stage, households at the second, and individuals within the target households at the third stage. Target households within the sample points were determined according to the random route procedure, target persons were selected according to random digits. Informed consent was considered to have been given when individuals agreed to complete the interview. In all, 5025 personal interviews were conducted, which reflects a response rate of $65.1 \%$. With regard to gender and age, the sample was comparable with the whole of the German population aged 18 years and older in 2000 (Statistisches Jahrbuch 2002/Official Registry Report 2002).

Interview

Vignette. The fully structured interview began with the presentation of a vignette containing a diagnostically unlabelled psychiatric case history. De facto, the case histories either depicted a case of schizophrenia or major depressive disorder. The symptoms described in the vignettes fulfilled the criteria of DSM-III-R for the respective disorder. Before being used in the surveys, the vignettes had been submitted to five psychiatrists or psychologists (all proven experts on psychopathology) for blind diagnostic allocation. All experts were able to provide the correct diagnoses for both case histories. Randomly drawn subsamples were presented with either the vignette with a case of schizophrenia or with that with a case of major depression.

Labelling. Using an open-ended question, respondents were asked to indicate how they would label the problem described in the vignette. The responses were noted down by the interviewers to be later coded using a coding system which already proved to be useful in previous surveys. Four main categories were distinguished: correct psychiatric diagnosis (e.g. 'schizophrenia', 'persecutory delusion', 'psychosis'); other psychiatric illness or psychiatric illness unspecified; personal problem (i.e. the subject described in the vignette is facing an acute life crisis or some chronic difficulty); other definitions of the problem depicted in the vignette. If multiple labels were suggested by the respondent, only the label that came closest to the correct psychiatric diagnosis was registered. Interrater reliability was checked by having two people code 200 interviews independently of one another. 
Cohen's kappa reached 0.85 . For our analyses, the first two categories were combined to the category 'labelling as mental illness'.

Personal attributes. Drawing on previous findings of stigma research a list of eight personal attributes was generated which was intended to cover two important components of the stereotype of mental illness: dangerousness and dependency. Respondents were asked to indicate, with the help of a fivepoint Likert scale, ranging from 'definitely true' (code 1) to 'definitely not true' (code 5), to what extent these attributes apply to the person depicted in the vignette. Confirmatory factor analysis yielded an acceptable fit for the two dimensions [Comparative Fit Index (CFI) 0.935, Tucker Lewis Index (TLI) 0.905 for a sample size of 4987 individuals]. The model chi-square is 946.6 at 19 degrees of freedom. Factor loadings and factor correlation are reported in Table 1. Factor determinacies are 0.945 for dangerousness and 0.821 for dependency, which is considered to be sufficient. Scoring was reversed on both factors to facilitate interpretation. Hence, high scores represent the endorsement of dangerousness as well as of dependency.

Emotional reactions. According to previous research (7), mainly three types of emotional reactions to people with mental illness can be distinguished: fear, pity, and anger. A list of 9 five-point Likertscaled $(1=$ definitely the case, $5=$ definitely not the case) items, representing these three ways to respond to mentally ill people, was used to assess respondents' emotional reactions to the individual described in the vignette. Confirmatory factor analysis yielded a fairly good fit for the threedimensions as indicated by a CFI of 0.953 and a TLI of 0.930 for the total sample $(n=4948)$. Chi square is 559.6 at 24 degrees of freedom. Table 2 shows the factor loadings and factor correlations. Factor determinacies are 0.909 for fear, 0.832 for pity, and 0.878 for anger. Scoring was reversed on

Table 1. Factor loadings and correlations for personal attributes

\begin{tabular}{lcc}
\hline & Loadings & $R^{2}$ \\
\hline Perceived dangerousness & & \\
$\quad$ Unpredictable & 0.779 & 0.606 \\
Lacking self control & 0.817 & 0.667 \\
Aggressive & 0.818 & 0.669 \\
Frightening & 0.673 & 0.453 \\
Dangerous & 0.801 & 0.641 \\
Perceived dependency & & \\
$\quad$ Needy & 0.321 & 0.103 \\
Dependent on others & 0.686 & 0.471 \\
$\quad$ Helpless & 0.677 & 0.458 \\
Factor correlation & 0.447 & \\
\hline
\end{tabular}

Table 2. Factor loadings and correlations for emotional reactions

\begin{tabular}{lcc}
\hline & Loadings & $R^{2}$ \\
\hline Fear & & \\
$\quad$ Fear & 0.783 & 0.613 \\
Uneasiness & 0.821 & 0.673 \\
Feelings of insecurity & 0.684 & 0.468 \\
Pity & & \\
$\quad$ Pity & 0.536 & 0.287 \\
$\quad$ Empathy & 0.536 & 0.287 \\
Desire to help & 0.765 & 0.585 \\
Anger & & \\
Anger & 0.779 & 0.607 \\
$\quad$ Ridicule & 0.566 & 0.321 \\
$\quad$ Irritation & 0.712 & 0.508 \\
Factor correlation & & \\
& Fear & \\
Pity & 0.028 & -0.181 \\
Anger & 0.512 & \\
\hline
\end{tabular}

all three factors to facilitate interpretation. Hence, high scores represent the tendency to react with fear, pity or anger, respectively.

Social distance. For the assessment of respondents' desire for social distance, we made use of a scale developed by Link (4), a modified version of the Bogardus Social Distance Scale (8). It includes seven items representing the following social relationships: rent a room, common place of work, neighbourhood, member of the same social circle, personal job brokering, marriage into one's family, child care. Using a five-point Likert scale ranging from 'in any case' (1) to 'in no case at all' (5), the respondents could indicate to what extent they would, in the situation presented, accept the person described in the vignette. Since the items chosen represent relationships of different degrees of intimacy which results in different item difficulties a factor analysis may lead to an artificial multidimensionality where each factor comprises items with similar difficulty. Therefore, a non-linear principal component analysis (9) was carried out. The first axis derived by this analysis proved to be sufficient to measure social distance. High scores indicate a preference for great social distance. The reliability of the scale, assessed by means of Cronbach's alpha, was 0.90 .

The interview was identical with the one which had already been applied in previous studies (e.g. $7,11)$. Therefore, no pretesting was necessary.

\section{Results}

A total of $70.8 \%$ of the respondents, who were presented with the vignette depicting a case of schizophrenia, labelled it as mental illness. Major 


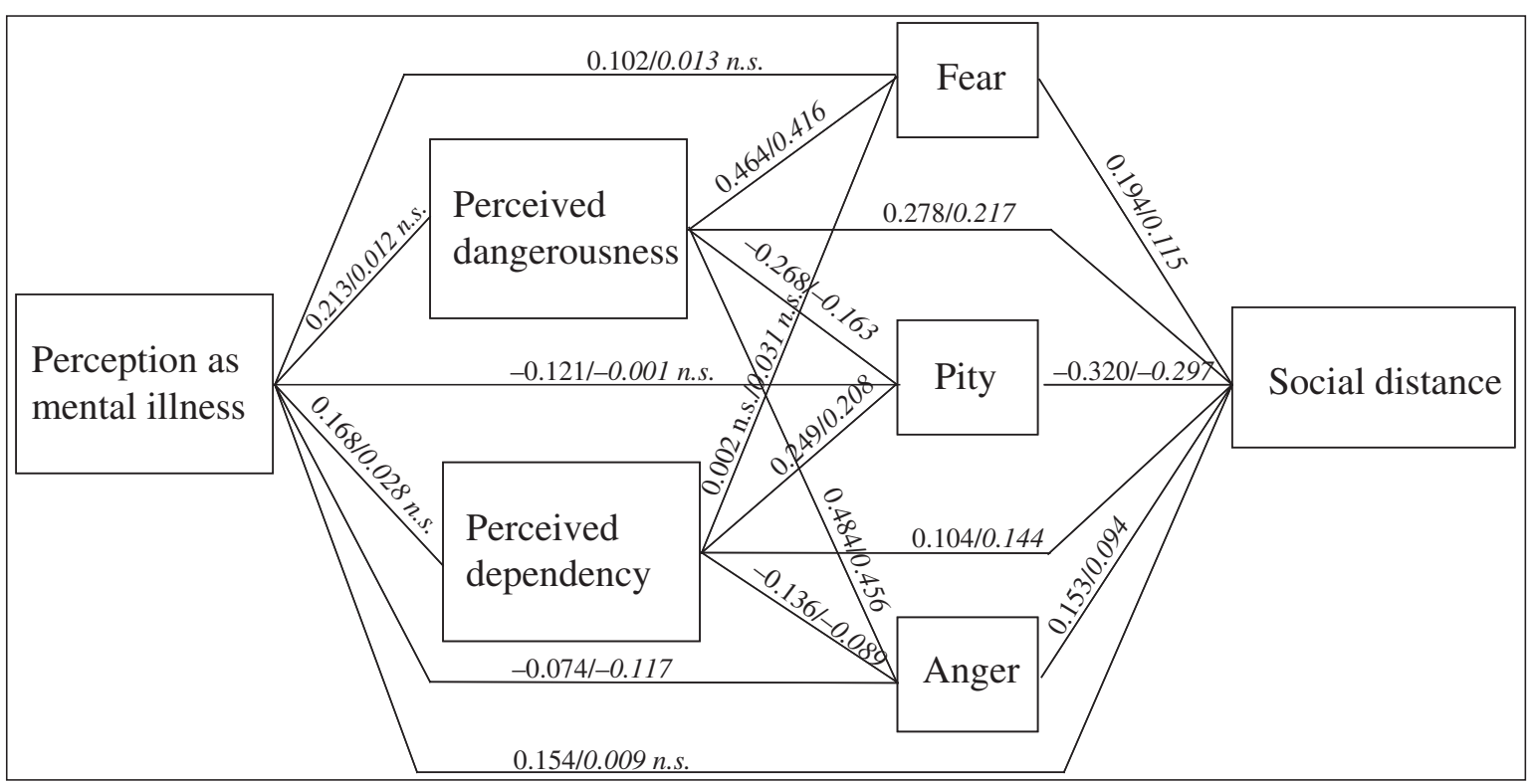

Fig. 1. Relationship between perception as mental illness, personal attributes, emotional reactions, and social distance: schizophrenia $(n=2378)$ vs. major depression $(n=2448)$ (unstandardised path coefficients; italics: major depression).

depression was slightly less frequently, by $62.0 \%$, identified as mental illness. Fig. 1 shows for both disorders the path model for the relationships between labelling as mental illness, personal attributes (perceived dangerousness, perceived dependency), emotional reactions (fear, pity, anger), and social distance. Instead of testing the measurement part and structural part of the model simultaneously, we used a two-stage approach (11) with confirmatory factor analysis and non-linear principal component analysis, respectively, being the first stage and path analysis being the second stage. The path model is estimated as a saturated linear regression model, fully recursive and without any restrictions.

As concerns schizophrenia, all path coefficients are significant. Respondents who label the individual depicted in the vignette as being mentally ill are more likely to believe that this individual is dangerous. The perception of dangerousness, in turn, is closely associated with fear and anger. In addition, there is also an inverse relationship with pity. As expected, a positive relationship exists between fear and social distance. Besides that, there is also a negative association between pity and the preference for social distance which is even stronger. The positive association of anger with social distance is by far the weakest. In addition, there is a direct positive relationship between perceived dangerousness and social distance: the more the individual in the vignette is perceived as dangerous, the more respondents express a preference for social distance. Labelling as mental illness is also positively associated with the other attribute ascribed to the individual suffering from schizophrenia: those who use the label of mental illness more frequently attribute features of dependency. Perceived dependency, in turn, has an effect on the three emotional reactions, which, however, is smaller than that of perceived dangerousness and somehow inconsistent: as expected, pity increases and anger decreases; the effect on fear can be neglected. The expression of anger is also directly affected by labelling: those who identify the individual as mentally ill are less ready to react with anger.

As already expected from the above-reported findings, the path coefficients for the relationships between labelling and attitudes towards the individual with major depression are not statistically significant, except for anger: respondents who perceive the individual as mentally ill are less prone to react with anger. As with schizophrenia, there is a close positive association between perceived dangerousness on one hand, and fear and anger as well as social distance on the other hand. There is also a close inverse relationship between pity and social distance. While with schizophrenia, the path model explains $26.7 \%$ of the variance of social distance, with major depression it explains only $18.9 \%$.

\section{Discussion}

Summarizing our findings we can state: as concerns schizophrenia, the hypotheses referring to the 
relationship between labelling on the one hand, and perceived dangerousness, fear, and social distance on the other hand are supported by our findings. Labelling as mental illness is positively correlated with the endorsement of the belief that the individual depicted in the vignette is dangerous (hypothesis 1). This, in turn, leads directly as well as indirectly through an increase of fear to a preference for greater social distance (hypotheses 2 and 3). Besides that, perceived dangerousness results in an increase of social distance through an inverse relationship with pity. By contrast, labelling is found to have no effect on these attitudinal components with major depression. There is neither a statistically significant association with perceived dangerousness, nor with fear or social distance.

As predicted in hypothesis 4, labelling the individual with schizophrenia as mentally ill is also positively associated with perceived dependency. However, the positive association of perceived dependency with pity as well as the negative association with anger, both predicted in hypothesis 5 , is less strong than that between perceived dangerousness and fear. There is also a positive correlation between perceived dependency and fear, i.e. it not only provokes pity and reduces anger, but also increases fear (which, in turn, leads to increased social distance). Thus, its effect on emotional reactions is rather contradictory. In addition, the association between anger and social distance hardly exceeds the significance level. This means that the reduction of anger does practically not translate into a decrease of the desire for social distance; this holds only true for pity (as predicted in hypothesis 6). Again, in the case of major depression, there are no statistically significant relationships, except for anger which is inversely associated with labelling. Hypothesis 7 is, thus, fully supported by our findings, even to a larger extent than one might have expected.

From these findings we can draw the following conclusions:

1) Labelling as mental illness has an impact on public attitudes towards people with schizophrenia, with negative effects clearly outweighing positive effects.

2) Endorsing the stereotype of dangerousness has a strong negative effect on the way people react emotionally to someone with schizophrenia and increases the preference for social distance.

3) By contrast, perceiving someone with schizophrenia as being in need for help evokes mixed feelings and positively as well as negatively affects people's desire for social distance both.
4) Labelling has practically no effect on public attitudes towards people with major depression.

What are the implications of our findings for interventions aimed at reducing the stigma of mental illness? As we have seen, the label of mental illness still matters. The price schizophrenia patients pay for disclosing their illness seems by far higher than the potential benefits. In view of this, it is not surprising that the majority of patients, when being ask about strategies for stigma coping, recommend to keep mental illness a secret or even to avoid contact with other people $(12,13)$. People who are identified as being mentally ill are at a high risk of being stigmatized. Particularly the linkage with the stereotype of dangerousness provokes adverse reactions among the public. Therefore, it should be the key target for anti-stigma interventions. Experimental studies suggest that among the available strategies, education, protest, and contact with a person with mental illness, the latter may be most effective in inducing change (14). Here again, interventions in schools facilitating encounters between students and people with schizophrenia appear particularly promising (15).

By contrast, labelling people with major depression as mentally ill appears to have no substantial impact on public attitudes. This may in part be due to the fact that the term 'depression' is somewhat enigmatic, denoting a wide range of mental health problems. Respondents who offered this 'diagnosis' may not necessarily have thought of a serious mental disorder, as it most likely has been the case with schizophrenia. As our findings also indicate, depression is more accepted by the general public than schizophrenia, with or without being labelled.

In conclusion, we hope to have been able to demonstrate the usefulness of the theoretical concept applied in this study. We want to emphasize the need for differentiation, differentiation between the different components of stigma (e.g. stereotype, prejudice, discrimination) as well as differentiation between the various mental disorders. This appears important not only on theoretical grounds. It may be even more important for practical reasons. Anti-stigma efforts may only prove successful if they take into account the complexity of the stigma process, and if they are tailored to impact those factors which are of main importance.

\section{Acknowledgement}

The project was supported by the German Research Association (grant AN 101/5-1). 


\section{References}

1. Parsons T. Definitions of health and illness in the light of American values and social structure. In: JACO EG, ed. Patients, physicians and illness. New York: Free Press, 1958.

2. Scheff TJ. Being mentally ill. Chicago: Aldine, 1966.

3. Farina A. Stigma. In: Mueser KT, TArrier N. Handbook of social functioning in schizophrenia. Boston: Allyn and Bacon, 1998:247-279.

4. Link BG, Cullen FT, Frank J, Wozniak JF. The social rejection of former mental patients: understanding why labels matter. Am J Sociol 1987;92:1461-1500.

5. Corrigan PW. Mental health stigma as social attribution: implications for research methods and attitude change. Clin Psychol Sci Prac 2000;7:48-67.

6. Corrigan PW, Watson AC. Understanding the impact of stigma on people with mental illness. World Psychiatry 2002;1:6-20.

7. Angermeyer MC, Matschinger H. Social distance towards the mentally ill: results of representative surveys in the Federal Republic of Germany. Psychol Med 1997;27:131-141.

8. Bogardus ES. Measuring social distances. J Appl Sociol 1925;1 \& 2:216-226.
9. GiFI A. Non-linear multivariate analysis. Chichester: Wiley, 1990.

10. Angermeyer MC, Matschinger H, Riedel-Heller SG. What to do about mental disorder-help-seeking recommendations of the lay public. Acta Psychiatr Scand 2001;103:220-225.

11. Anderson J, Gerbing DW. Structural equation modelling in practice: a review and recommended two-step approach. Psychol Bull 1988;103:411-423.

12. Holzinger A, Beck M, Munk I, Weithaas S, Angermeyer MC. Das Stigma psychischer Krankheit aus der Sicht schizophren und depressiv Erkrankter. Psychiat Prax in press.

13. Link BG, Struening EL, Rahav M, Phelan JCC, Nuttbrock L. On stigma and its consequences: evidence from a longitudinal study on men with dual diagnoses of mental illness and substance abuse. J Health Soc Behav 1997;38:177-190.

14. Corrigan PW, Rowan D, Green A, Lundin R, River P, UphoffWasowski K, White K. Challenging two mental illness stigmas: personal responsibility and dangerousness. Schizophr Bull 2002;28:293-309.

15. Schulze B, Richter-Werling M, Matschinger H, Angermeyer MC. Crazy? So what!? Project weeks on mental health and illness. Their effect on secondary school students' attitudes towards people with schizophrenia. Acta Psychiatr Scand 2003; 107:142-150. 